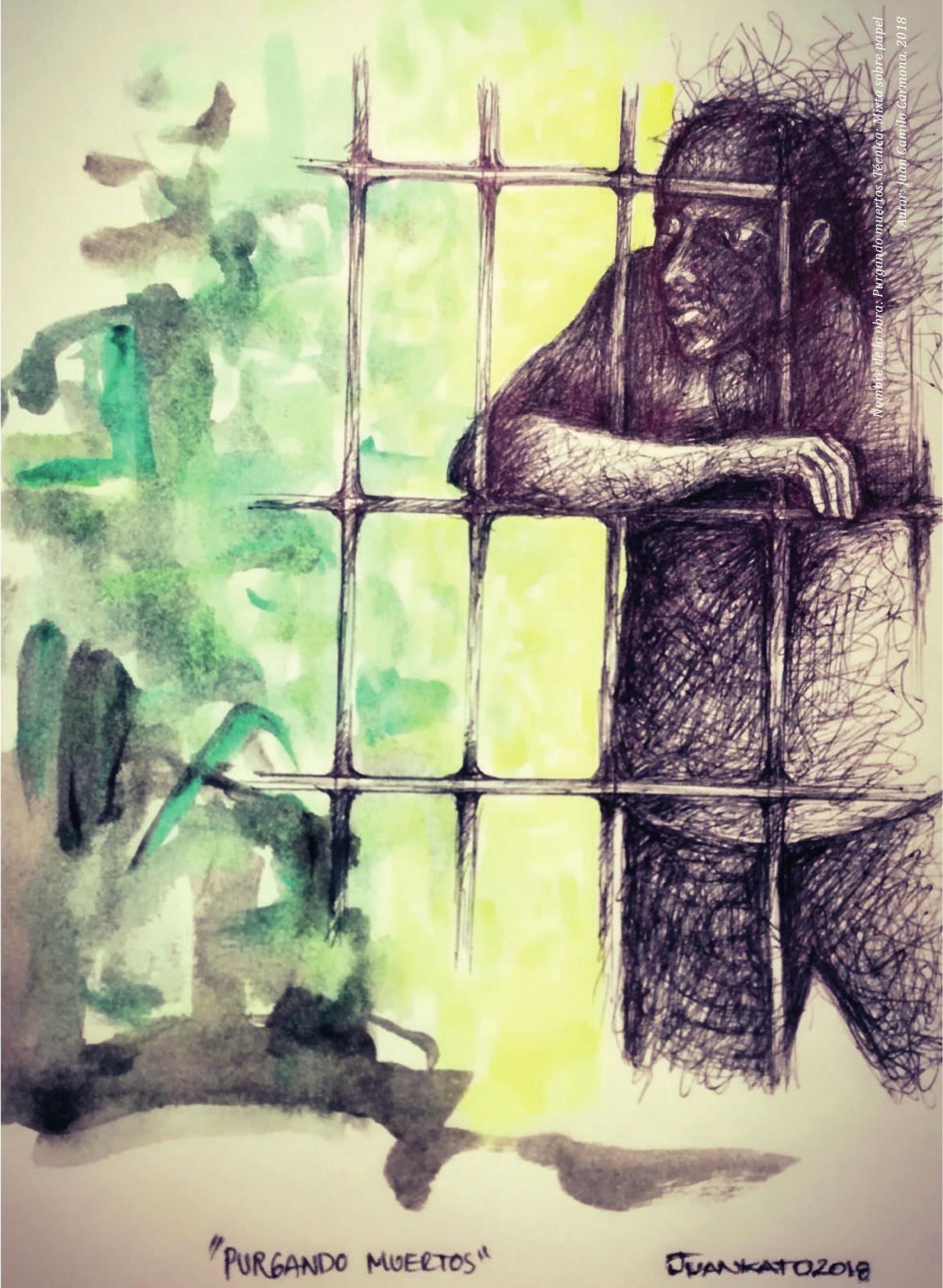




\title{
La seguridad después de la guerra: nuevos referentes de seguridad para el post acuerdo de paz en Colombia*
}

\author{
Ángela Navia López**
}

\section{Resumen}

Los acuerdos de paz de La Habana presentan una visión moderna y cualitativamente nueva de seguridad. Aluden a la seguridad humana como un derecho de la sociedad. Sin embrago, la noción de seguridad no es unívoca. En Colombia ha persistido la visión clásica de seguridad reflejada en la Doctrina de Seguridad Nacional. El presente artículo analiza esta doctrina y argumenta la necesidad de transitar a otros paradigmas de seguridad, como la seguridad humana, como requisito para la construcción de paz. En primer lugar, se realiza un acercamiento a la conceptualización de seguridad. Posteriormente, se describen las principales características de la Doctrina de Seguridad Nacional, evidenciando su evolución histórica y lo imperioso de su abandono. Esto permite, finalmente, resaltar la importancia de la concepción de seguridad humana erigida en los acuerdos de paz.

Palabras clave: acuerdos de paz; seguridad; doctrina de seguridad nacional; seguridad humana.

\section{The Security after the war: new security referents for the post-peace agreement in Colombia.}

\begin{abstract}
The Habana peace agreements present a modern and qualitatively new vision of security. Specifically, they refer to human security as a right of society. However, the notion of security is not univocal. In Colombia, the classic vision of security reflected in the National Security Doctrine has persisted. This article analyzes this doctrine and argues the need to move to other security paradigms, such as human security, as a prerequisite for the construction of peace. In the first place, an approach to the conceptualization of security is made. Subsequently, the main characteristics of the National Security Doctrine are described, evidencing its historical evolution and the imperative of its abandonment. This allows, finally, to highlight the importance of the conception of human security established in the peace agreements.
\end{abstract}

Keywords : Peace Agreements; Security; Doctrine of national security; human security.

\section{A segurança depois da guerra: novos referentes de segurança para o pós-acordo de paz na Colômbia}

\section{Resumo}

Os acordos de paz de Havana apresentam uma visão moderna e qualitativamente nova de segurança. Aludem à segurança humana como um direito da sociedade. No entanto, a noção de segurança não é unívoca. Na Colômbia persiste a visão clássica de segurançar refletida na Doutrina de Segurança Nacional. Este artigo analisa esta doutrina e argumenta anecessidade de transitar a outros paradigmas de segurança, como as segurança humana, como requi sito para a construção da paz. Em primeiro lugar, realiza-se uma aproximação da conceptualização de segurança. Posteriormente, descrevem-se as principais características da Doutrina de Segurança Nacional, tornando evidente a sua evolução histórica e o caráter imperioso do seu abandono. Isto permite, finalmente, salientar a importância da concepção de segurança humana erigida nos acordos de paz.

Palavras-chave: acordos de paz; segurança; doutrina de segurança nacional; segurança humana.

\footnotetext{
*Artículo de reflexión. Es derivado del proyecto en curso Seguridad Humana en contextos de Justicia Transicional. Estudio para el Departamento de Nariño. El proyecto se adscribe al grupo de investigación Saber Sociojurídico, en la línea de investigación "Problemas contemporáneos de la filosofía y de la teoría del derecho". Grupo de investigación de la Universidad Mariana. Investigadora principal: Ángela Navia. El proyecto se encuentra en curso, finaliza en diciembre de 2018.

** Docente-investigadora de la Maestría en Derecho de La Universidad Mariana, Colombia. Abogada de la Universidad de Nariño. Magister en Relaciones Internacionales con mención en Seguridad y Derechos Humanos de la Facultad Latinoamericana de Ciencias Sociales - FLACSO. Integrante del grupo de investigación Saber Sociojurídico. Correo electrónico: angela_navi@hotmail.com ORCID:0000-0002-0640-8037
}

Cómo citar este artículo: Navia, Á. (2019). La seguridad después de la guerra: nuevos referentes de seguridad para el post acuerdo de paz en Colombia. Estudios de Derecho, 76 (167), pp-pp.93-112

DOI: 10.17533/udea.esde.v76n167a04

Fecha de recepción: 15/03/2018 Fecha de aprobación: 21/06/2018 


\section{La seguridad después de la guerra: nuevos referentes de seguridad para el post acuerdo de paz en Colombia}

\section{Introducción}

Los acuerdos de paz de La Habana presentan una visión moderna y cualitativamente nueva de seguridad. Su preámbulo reconoce los derechos de la sociedad a una seguridad humana integral con participación de las autoridades civiles. Dicho reconocimiento pareciera ser una declaración elemental y de sentido común en un acuerdo de paz, en la medida que hablar de paz implica posicionar las dimensiones humanas antes que las militares. Sin embargo, en Colombia no resulta evidente aludir a la Seguridad Humana cuando históricamente la concepción de seguridad ha sido militarizada, es decir, cuando se reduce la noción de seguridad a temas militares.

La militarización de la noción de seguridad atendió a la lógica de la guerra fría. Hobsbawm (2007) advierte que un requisito indispensable para culminar los conflictos armados en el siglo XXI consiste en superar tal noción que surge asociada al conflicto entre EEUU y la antigua Unión Soviética. Atendiendo este corolario, para que Colombia pueda transitar hacía un escenario de paz en el post acuerdo es necesario abandonar aquella concepción tradicional de seguridad.

En este sentido, el presente artículo pretende argumentar que la construcción de paz necesita nuevos referentes de seguridad. En específico, se busca analizar las dos concepciones de seguridad, la que ha existido en Colombia y la referida en los acuerdos de La Habana. Las dos enmarcadas en contextos internacionales históricos y sustentados en paradigmas teóricos específicos. A partir de este análisis se busca comprender y dimensionar la noción de seguridad humana como un nuevo referente de seguridad.

Para tal fin el trabajo se compone de cuatro acápites. En el primero se realiza un acercamiento conceptual a la noción de seguridad. Para ello se acude a los estudios de seguridad que ha se han desarrollado desde la teoría de las Relaciones Internacionales. Desde aquí se evidencia el momento histórico y teórico de los dos referentes de seguridad, así como el viraje en los paradigmas de seguridad. Establecido lo anterior, en segundo momento, se analiza la visión clásica de segu- 
ridad como el referente predominante en Colombia. En específico, se examinan las principales características de la Doctrina de Seguridad Nacional -DSN-, se realiza un recuento histórico y se observan sus impactos. El tercer acápite se ocupa del otro referente de seguridad, la seguridad humana, se estudia su surgimiento y características. Igualmente, se analiza esta noción de seguridad en clave de construcción de paz. Esto permite, finalmente, erigir conclusiones sobre los dos referentes de seguridad, lo que ha implicado la adopción de la DSN y la importancia de la seguridad humana para transitar hacia la paz.

\section{Seguridad: conceptualización y debates teóricos}

La Real Academia de la Lengua Española define seguridad como la cualidad de estar seguro, es decir, estar libre y exento de riesgo. La seguridad existe cuando se presenta una amenaza, cuando algo o alguien está en peligro y, por tanto, deben emprenderse las medidas para ser cuidado. En una definición ajustada puede decirse que la seguridad consiste en librarse de las amenazas y en ser capaz, bien sean los estados o las sociedades, de mantener su independencia en lo que se refiere a su identidad, y a su integración funcional, frente a fuerzas de cambio consideradas hostiles (Orozco, 2006, p. 169).

Explica Wæver que "antes del siglo XX, la seguridad no era un concepto clave o el eje del pensamiento internacional. Un primer paso en esta dirección vino con la seguridad colectiva en el periodo entre guerras" (2009, p. 78). Desde entonces las potenciales mundiales empezaron a usar la seguridad como lema de su política exterior. Por esto, la seguridad se convierte en objeto de estudio de las diferentes disciplinas de las Ciencias Sociales, en especial en las Relaciones Internacionales.

Sin embargo, durante la guerra fría las categorías dominantes para analizar el sistema internacional fueron el poder y los estudios de paz. La seguridad se encontraba inmersa en ellos. Los impactos que implicó el fin orden mundial bipolar generaron la pérdida de capacidad explicativa las dos categorías hasta entonces predominantes. Emerge, entonces, la seguridad como una categoría de análisis que abarca mayores elementos para estudiar las dinámicas del nuevo orden mundial (Orozco, 2006).

En los estudios sobre seguridad se deben identificar dos elementos centrales: 1) el objeto referente y 2) su contenido (Orozco, 2006). El primero responde a la pregunta ¿qué debe protegerse? Es decir, es el objeto que se debe resguardar. El contenido del objeto referente es lo que se encuentra amenazado y, por tanto, es el fin a proteger. Al establecer estos elementos, lo que se determina en últimas es, en términos de Møller (1996), de quién es la seguridad.

Determinar el objeto referente dependerá de la teoría desde donde se aborde. Así, por ejemplo, para el realismo, visión que posiciona al Estado como actor 
principal del Sistema Internacional y al poder como su elemento de búsqueda (Morgenthau, 1986), el objeto referente es el Estado y el contenido a preservar es la integridad territorial y la soberanía. Desde esta corriente el objetivo de la política de seguridad debe ser "disponer de todos los medios indispensables, así como de los recursos necesarios para preservar el interés de la nación, pues con ello se logrará mantener la integridad y librar de las amenazas a aquellos intereses prioritarios del Estado" (Orozco, 2006, p.166).

Por su parte, para el constructivismo, teoría que sitúa como actor principal del sistema internacional a los agentes o grupos sociales (Wendt,1992), el objeto referente es las colectividades y su contenido, lo que se debe preservar, es la identidad de las comunidades.

Ahora bien, determinar lo que se debe preservar -objeto referente- y de qué se debe resguardar -la amenaza- no resulta una actividad aislada e improvisada. Es una acción que refleja los intereses de las clases dominantes en un determinado contexto histórico. Se trata de una determinación que tendrá implicaciones en las políticas internacionales y doméstica de los Estados.

Orozco (2006) propone, como herramienta metodológica para analizar la seguridad, la diferenciación entre dos modelos o referentes de seguridad: visión restringida de seguridad y visión amplia. La primera "se encuadra en los paradigmas que han elaborado modelos clásicos de seguridad, en tanto que en la segunda se abordan fenómenos emergentes del final de la Guerra Fría y de la actual condición de globalización" (p.170). Es decir, la visión clásica responde, en términos teóricos, a la visión realista y, en términos históricos, se enmarca en el periodo de la guerra fría. Por su parte el paradigma que ensancha los objetos referentes de seguridad-donde se ubica la seguridad humana- se adscribe a teorías críticas de las Relaciones Internacionales y emerge en el escenario posguerra fría.

En este orden de ideas, durante la guerra fría predominó el enfoque realista, posicionando una visión clásica de seguridad o, dicho de otro modo, un referente estado céntrico en la medida que el objeto referente y principal preocupación era el Estado. El contexto histórico bipolar determinó que el mecanismo para proteger el Estado era la militarización.

Explica Eric Hobsbawm (1994) que en los primeros años de la guerra fría no existió una amenaza latente que desbocara en una confrontación directa, esto por un acuerdo tácito de repartir las ganancias de las dos potencias. Por eso, el autor plantea que hasta mediados de la década del setenta existió una especie de "paz fría" (Hobsbawm, 1994, p. 232). Esa clase de paz, paradójicamente, fue resultado de una seguridad clásica, sustentada en la militarización. A mayor fuerza militar, menor posibilidad de ataque por parte de otro Estado. Por esto, uno de los efectos de la guerra fría, explica Hosbawm, fue la militarización: "la guerra fría había llenado el mundo de armas hasta un punto que cuesta creer. Ese fue el resultado 
natural de cuarenta años de competencia constante entre los grandes estados industriales por amarse a sí mismo" (Hobsbawm, 1996, p. 257).

Si bien el aspecto más visible de la guerra fría fue la militarización, este no fue el principal impacto, en la medida que las armas no se usaron a nivel global. El principal impacto fue político (Hobsbawm, 1996, p. 241). En esta lógica, EEUU necesitaba mantener su accionar con el argumento de la existencia de un enemigo peligroso, no solo en términos militares, también como amenaza política, social e incluso cultural. Ese enemigo fue el comunismo ${ }^{1}$ :

El enemigo es el sistema comunista en sí: implacable, insaciable, infatigable en su pugna por dominar el mundo... Esta no es una lucha sólo por la supremacía armamentística. También es una lucha por la supremacía entre ideologías opuestas: la libertad bajo un Dios, y una tirana atea. (Walker, 1993 p. 132. Citado por Hobsbawm 1995 p. 235)

El posicionamiento de este enemigo a escala global va a hacer determinante de la DSN que se aplicará a los países de América Latina. Así como un detonante para el conflicto interno en Colombia. En este orden de ideas, el fin de la Guerra Fría "suprimió de repente los puntales que habían sostenido la estructura internacional hasta entonces" (Hobsbawm, 1996, p. 285). Lo que implicó, entre otras cosas, la emergencia de nuevas amenazas, necesidades y demandas del nuevo mundo globalizante. Esto hizo repensar la seguridad.

Entre 1947 y 1989 la seguridad nacional y militar “dominó en el discurso científico y se había convertido en medios (armas), instrumentos (inteligencia) y estrategias (disuasión)" (Oswal \& Gunter, 2010, p.46). Esta situación ha generado, incluso hasta la fecha, que los estudios sobre seguridad presenten dos problemas: la restricción de la seguridad a los debates sobre la seguridad nacional y su reducción a temas exclusivamente militares. Lo segundo desembocó en la idea de que "la respuesta a los problemas de inseguridad era exclusivamente fortalecer el tamaño de las Fuerzas Armadas y organismos de seguridad del Estado y aumentar el gasto militar en detrimento del gasto social o de inversión" (Vargas, 2010, p. 16).

A finales de la década de 1980 se empezó a disputar ese dominio. Se introdujeron en el debate nuevas corrientes y sectores que cuestionaron el objeto referente hasta entonces dominante. Desde entonces, "la seguridad se volvió un campo de batalla. El así llamado debate de la ampliación del concepto de seguridad se refiere a una batalla académica (...). No obstante, el debate fue antes que nada una lucha política" (Wæver, 2010, p. 90). Determinar nuevos objetos referentes implicaba pensar y repensar modelos económicos, políticos y sociales, que pudieran ser la

1 Esta connotación de enemigo será exportada a los países latinoamericanos, como se explicará en el siguiente acápite, e implicará para Colombia atizar la génesis y desarrollo de un prologando conflicto armado. 
causa de las nuevas amenazas. Wæver (2010) explica esto con el ejemplo de los problemas ecológicos o ambientales. Posicionar al ambiente como objeto referente, implicaba determinar las amenazas y ello conlleva a debatir políticamente los impactos de la actividad económica sobre la naturaleza. Luego, no ha sido fácil posicionar sobre la agenda de los países la noción de seguridad ambiental.

En este debate se da el quiebre histórico o cambio de paradigmas de seguridad, de la clásica a visiones críticas. Se empezaron a posicionar en el debate nuevas amenazas y preocupaciones. En este viraje de paradigmas juega un papel determinante la Escuela de Copenhague. Se trata del conjunto de pensadores (Buzan, Moller, Olewer, entre otros) que trabajaron en el Instituto de investigaciones para la paz. La escuela asume el tema de seguridad intentando unir dos posiciones encontradas: el realismo y el idealismo que se sustenta la idea kantiana ${ }^{2}$ de un orden mundial en paz. Para ello plantean la necesidad de expandir, no extinguir, la noción de seguridad.

Es por esto que la Escuela de Copenhague se convierte en el puente para las teorías críticas de seguridad y para lo que emergerá como seguridad humana. Esto por tres razones: primero, Orozco (2006a) señala como una característica de esta escuela que sus análisis emergen desde paradigmas de pensamiento europeo. Circunstancia que va a resultar importante, explica Orozco (2006a), para empezar a fracturar el paradigma tradicional de la seguridad, sustentado, principalmente, en escuelas norteamericanas. No se trata únicamente de la posición geográfica; se trata que de aquel lado del hemisferio la preocupación central no se deriva, a diferencia de la estadounidense, de la tensión con la Unión Soviética. Ello va a generar agendas disimiles en el análisis.

Segunda, la escuela va llamar la atención sobre la determinación del objeto referente. Advierte que el Estado no puede ser el único sujeto de la seguridad y las implicaciones que esto tiene. Es por ello que los estudios que emergen desde esta escuela van a posicionar nuevos objetos referentes derivados de las problemáticas emergentes de la posguerra fría (Orozco, 2006a, p.150).

Tercera, la Escuela de Copenhague asume que el contenido del objeto referente no es un solo elemento. Por el contrario, se trata de un contenido multidimensional. Así, por ejemplo, para la visión estado céntrica de la seguridad, las amenazas no solo recaen sobre la soberanía; también deben preservarse la democracia, el régimen político, la población, como uno de los componentes del Estado. Para la seguridad humana, que posiciona al ser humano como objeto referente, el contenido es todas las dimensiones que integran la dignidad del individuo. De ahí que

2 Esta corriente se sustenta, entre otras obras, en la obra de de Kant de 1975 La Paz Perpetua, en la cual plantea la noción de que es posible un orden mundial y una forma de gobierno que promuevan la paz. De ahí que acogen la importancia de las Instituciones Internacionales como mecanismos para resguardar la paz mundial. 
el factor multidimensional va a hacer una de las características fundamentales de la seguridad humana.

En síntesis, en el nuevo orden mundial emergen nuevos paradigmas de seguridad que amplían la concepción clásica. Entre ellos la seguridad humana. A la par se posiciona la importancia de los derechos humanos como elementos que garantizan contextos de democracia y paz al interior de los Estados. Antes de abordar el tema de seguridad humana es necesario comprender la influencia del paradigma clásico de seguridad en Colombia.

\section{Visión clásica de seguridad: un paradigma predominante en Colombia}

En Colombia la visión clásica de seguridad ha sido el paradigma predominante. La génesis y dinámica del conflicto armado, así como los intentos para su solución han estado determinados por la aplicación de esta política de seguridad. Para los países latinoamericanos, en general, la materialización de esa visión clásica de seguridad se condensa en lo que se conoce como la Doctrina de Seguridad Nacional -DSN-.

\section{Elementos generales de la DSN}

El Estado de seguridad nacional, concepción elevada después de la segunda guerra mundial en Europa occidental y EEUU, como alusión a la defensa militar de amenazas externas, tuvo su variante para América Latina en la Doctrina de Seguridad Nacional. Se trata de «una concepción militar del Estado y del funcionamiento de la sociedad, que explica la importancia de la "ocupación" de las instituciones estatales por parte de los militares». (Leal,2003, p.75). Explica Leal (2006) que esta doctrina ha sido el mayor esfuerzo de América Latina por militarizar el concepto de seguridad (2006, p.26).

La DSN se resume en dos factores: primero, la concepción de que a partir de la seguridad del Estado se garantiza la de la sociedad, considerando que para lograr este objetivo era menester el control militar del Estado (Leal, 2006, p.6). Segundo, la sustitución del enemigo externo o clásico por el enemigo interno; de esta forma los "estados Latinoamericanos debían enfrentar al enemigo interno, materializado en supuestos agentes locales del comunismo. Además de las guerrillas, el enemigo interno podía ser cualquier persona, grupo o institución nacional que tuviera ideas opuestas a las de los gobiernos militares” (Leal, 2003, p.75).

Una traducción de enemigo interno que se sintetizó en cualquier expresión que se considere de corte comunista, lo que implicó que "cualquier opositor o crítico al régimen era una seria amenaza a los valores políticos trascendentales que conformaban y caracterizaban la nación, por lo que cualquier respuesta a esta situación fue considerada de legítima defensa" (Jiménez, 2009, p.80). 
De esta manera, la DSN instituyó la militarización del concepto seguridad. Se hizo de ella un sentido común: el Estado Nación, en uso del poder coercitivo, debe garantizar el orden a la sociedad, el cual se asegura combatiendo al enemigo interno. La identificación de la amenaza (enemigo interno) desató una esquizofrenia en las instituciones castrenses: todo problema social se consideró subversivo. Se asumió al adversario político como antagónico que debe ser combatido militarmente.

Desde el punto de vista filosófico y de las Ciencias Políticas, las principales características de la DSN -fuerza del Estado mediante el componente militar y la determinación del enemigo interno-, pueden sustentarse desde dos autores: Thomas Hobbes y Carl Schmitt. La propuesta del contrato social de Hobbes, determinada en un Estado fuerte (Leviatán), se sustenta en la premisa de que únicamente mediante esta figura se controla el estado natural de los seres humanos y, de esta forma, puede alcanzarse una convivencia pacífica. Esto porque "la racionalidad presente en los hombres, de ninguna manera es suficiente para alcanzar la condición pacífica de vida que los hombres desean, siendo así necesario establecer pactos que estén respaldados siempre por la fuerza de la espada" (Ramírez, 2010, p.56). Se trata de la necesidad de un Estado fuerte como garantía para la convivencia y la paz.

Ahora bien, el concepto de seguridad en Hobbes "no se restringe únicamente a la garantía de la existencia física, sino que va más allá y se extiende, también, a la estabilidad social que permite disfrutar de una vida libre de amenazas" (Orozco, 2003, p. 164). Estas amenazas, puede inferirse de la obra de Hobbes, devienen principalmente del estado natural de los hombres, de ahí que es necesario, para estar seguros, el pacto social.

Adicional a estos sustentos que brinda el pensador inglés para la concepción de un Estado fuerte como garantía de paz, se encuentra la noción de que es el Estado, mediante la figura del soberano, el que puede determinar que es bueno o malo para la sociedad, situación que, bajo la óptica de Hobbes, no se da en la condición anárquica o natural de los hombres:

En la condición natural no existen los conceptos de bueno y malo, tuyo y mío, entre otros. La medida de lo bueno o de lo justo dependerå solamente de un criterio de utilidad que cada hombre particular ha de juzgar, lo que implica que nada por sí mismo ha de considerarse como bueno o como malo. (...) Una vez creado el Estado se abandona este relativismo para dar paso a un absolutismo moral, el cual es establecido a partir del criterio del soberano. El soberano tiene el poder de legislar, el cual consiste no solo en prescribir normas, sino también en establecer qué se ha de entender por bueno y malo, verdadero y falso, legitimo e ilegitimo, moral e inmoral. (Ramírez, 2010, p.66)

La DSN plantea la necesidad de un soberano que debe ser lo suficientemente fuerte, en especial, desde el aspecto militar o de coacción, para brindar seguridad a 
la sociedad. Este soberano, a su vez, es quien determina lo que es seguro o riesgoso $\mathrm{y}$, por tanto, emprende las acciones necesarias para mantener una sociedad libre de amenazas.

Por su parte, los planteamientos del alemán Carl Schmitt sirven de sustento explicativo para la noción de enemigo interno. Para Schmitt la esfera política se sustenta en la dicotomía amigo /enemigo y en la actividad de determinarlos o diferenciarlos. Es en esta actividad donde toma vida el quehacer político; circunstancia que diferencia la política de la moral. Mientras la última se ocupa de la trasposición bien y mal; la estética, por su parte, de lo bello y lo feo, la esencia de la política es amigo/enemigo. En palabras del autor:

La diferenciación específicamente política, con la cual se pueden relacionar los actos y las motivaciones políticas, es la diferenciación entre el amigo y el enemigo. Esta diferenciación ofrece una definición conceptual, entendida en el sentido de un criterio y no como una definición exhaustiva ni como una expresión de contenidos. En la medida en que no es derivable de otros criterios, representa para lo político el mismo criterio relativamente autónomo de otras (..) La diferenciación entre amigos y enemigos tiene el sentido de expresar el máximo grado de intensidad de un vínculo o de una separación, una asociación o una disociación. Puede existir de modo teórico o de modo practico, sin que por ello y simultáneamente todas las demás diferenciaciones morales, estéticas, económicas, o de otra índole, deban ser de aplicación. (Schmitt, 2002, p.56-57)

Advierte el autor alemán que el enemigo que sustenta la acción política debe ser de carácter público y no privado. Toda vez que su acción se relaciona con todo un pueblo. Por esto, argumenta Schmitt, debe combatirse. El autor no escatima esfuerzos en señalar que lo que se ha determinado como enemigo se convierte en un "otro", un diferente, que no requiere, expresa Schmitt, ser moralmente malo o estéticamente feo, "(e)s simplemente el otro, el extraño, y le basta a su esencia el constituir algo distinto y diferente en un sentido existencial" (Schmitt,2002, p.65).

Se trata de un antagónico con condición de alteridad pública. Contenido propio de la noción de enemigo interno. La DSN lo posiciona como un otro-extraño y amenazante que debe ser contrarrestado o eliminado para preservar la paz. Esta dicotomía, capaz de producir alteridad, crea en la población imaginarios y estigmas de tal magnitud que produce una simbología cultural que termina legitimando expresiones de violencia o extinción física de lo que se ha reconocido como enemigo. Esto podría explicar la violencia cultural desarrollada por el noruego Johan Galtung. Para él la violencia cultural son 
aquellos aspectos de la cultura, la esfera simbólica de nuestra existencia -materializado en la religión y la ideología, en el lenguaje y el arte, en la ciencia empírica y la ciencia formal (la lógica, las matemáticas)- que puede ser utilizada para justificar o legitimar la violencia directa o la violencia estructural. (Galtung, 2016, p. 149)

Luego, construir paz demanda, entre otros factores, eliminar la violencia cultural. Lo que implica abolir la dicotomía amigo/enemigo propio de la DSN.

\section{Desarrollo de la DSN en Colombia}

Francisco Leal Buitrago (2006) analiza el surgimiento de la DSN en cuatro etapas: antecedentes, gestación, desarrollo y declinación. La primera se posiciona en el siglo XIX en lo que se denomina viejo militarismo, es decir en las instituciones castrenses luego de la independencia. Su desarrollo va a la par de la consolidación de los estados nacionales; con la característica que estas instituciones fueron más fuertes que las otras instancias del Estado. Esta situación permitió que las instituciones militares se "autoproclamaran como dinamizadores de la economía e integradores de la sociedad" (2006, p. 31). Ello les dio un posicionamiento determinante en las decisiones y rumbo del país. Sin embargo, "los procesos de cambio en las Fuerzas Armadas hacia su conversión en instituciones profesionales modernas, van a estar íntimamente ligados a la evolución de los problemas de seguridad interior" (Vargas, 2009, p. 46).

La gestación se ubica en la guerra fría y responde a la influencia política, militar e ideológica de Estados Unidos. El acta de seguridad de 1947, la cual crea el Consejo de Seguridad Nacional (NSC) y la agencia central de inteligencia (CIA), fue un insumo para exportar la doctrina a los países del contiene. Esta se reforzó con el surgimiento, en el mismo año, del Tratado Interamericano de Asistencia Recíproca TIAR. Acuerdo clave para lograr cohesión de las instituciones militares en el continente. Adicional, EEUU se encargó de alimentar la DSN mediante la realización de conferencias anuales dirigidas a los comandantes de los Ejércitos del continente, las cuales se realizaron de manera ininterrumpida desde 1961 hasta inicios de 1980 (Leal, 2006, p. 61).

En esta etapa, la DSN también se nutrió de los principios franceses sobre seguridad desarrollados en la década del cincuenta y setenta para confrontar los movimientos de independencia de Indochina y Argelia. Se trata de una doctrina de seguridad colonialista puesto que el objetivo principal era mantener las colonias francesas. Sus creadores gestaron una doctrina antisubversiva. Entre sus principales características se resalta la guerra psicológica y la tortura, "en sus textos intentaron justificarla aduciendo su utilidad práctica como fuente de información, para salvar vidas y evitar nuevos ataques" (Vergara, 2005, p. 205). 
De esta forma las guerras antisubversivas son integrales (Zelik, 2011) puesto que no se ocupan únicamente del combate militar. También deben preocuparse y ocuparse de la cooptación de la población civil en zonas de influencia de los grupos subversivos. A la vez que usan las operaciones psicológicas como herramienta estratégica para la lucha contra el enemigo interno. Explica Zelik (2011) que estas operaciones son "medidas capaces de influir deliberadamente en los sentimientos y convicciones sociales de un grupo poblacional. Son reconocidas como un ingrediente esencial de la doctrina militar y de seguridad de los Estados Unidos" (p.178).

La tercera etapa, el desarrollo, Leal la posiciona desde el triunfo de la Revolución Cubana. Momento en el cual urge la implementación de esta doctrina en los países de Centro y Sur América. No obstante, sucesos en Colombia denotan el desarrollo o puesta en marcha de la doctrina desde los albores de la década del cincuenta, antes del surgimiento de las insurgencias como estructuras guerrilleras. Esto se lo puede corroborar con dos ejemplos: 1) la expedición del acto legislativo número 6 del 14 de septiembre de $1954^{3}$ por parte de la Asamblea Nacional Constituyente mediante el cual se prohíbe el "comunismo internacional". Regulación que permite inferir la asimilación de la lógica de identificación de un enemigo con sus respectivas medidas para menguarlo. 2) La acción de la fuerza pública el 8 y 9 de junio de $1954^{4}$ frente a la manifestación estudiantil en Bogotá, materializan la lógica de asumir acciones y expresiones sociales como combate al enemigo en nombre de la seguridad para la sociedad.

Años más adelante, en pleno desarrollo de la DSN, se expide el Decreto 1923 de 1978 mediante el cual se crea el Estatuto de Seguridad en el gobierno de Turbay Ayala. Expedición que fue posible por el decreto número 2131 de 1976 que declara el Estado de sitio en todo el territorio nacional. El Estatuto de Seguridad establece que "es indispensable adoptar medidas de seguridad para mantener el orden social y la paz en el territorio de la República". Afirmación que permite corroborar la visión de que al garantizar la seguridad del Estado se protege a toda la sociedad. Arrogándose, desde las esferas gubernamentales y de poder, la determinación de lo que es y no para el pleno de la sociedad.

Un ejemplo de la asimilación de los principios de DSN, referidos al control de lo que se entiende por orden público y seguridad, por parte de este Estatuto se ubica en el artículo 13, el cual establecía:

3 La norma puede ser consultada en http://www.suin-juriscol.gov.co/clp/contenidos.dll/Acto/1825725?fn=documentframe.htm $\$ \mathrm{f}=$ templates $\$ 3.0$

4 Marchas estudiantiles realizadas en memoria de Gonzalo Bravo Pérez, estudiante asesinado en 1929, las cuales obtuvieron como respuesta disparos por parte del Batallón Colombia, dejando un saldo de nueve muertos y varios heridos. 
Artículo 13. Mientras subsista la perturbación del orden público no podrán transmitirse por las estaciones de radiodifusión y por los canales de televisión informaciones, declaraciones, comunicados o comentarios relativos al orden público, al cese de actividades o paros o huelgas ilegales o noticias que inciten al delito o hagan su apología.

Esta disposición permite inferir la asimilación de las expresiones sociales, como las huelgas, a elementos delictivos y con potencial riesgo para la sociedad; es decir, como expresiones de un enemigo interno que puede poner en riesgo la estabilidad estatal.

Advierten Leal (2006) y Jiménez (2009) que el Estatuto de Seguridad Nacional les permitió a las instituciones militares acrecentar su autonomía en los asuntos de orden público y ampliar sus competencias e influencia en el poder ejecutivo.

Con la ampliación de sus funciones y la difusa frontera entre la influencia del poder civil y militar, el Estatuto de Seguridad aumentó las atribuciones de los comandantes del Ejército, institucionalizando nuevas competencias, entre ellas el juzgamiento de delitos y el control de manifestaciones, huelgas, paros cívicos y otro tipo de acciones colectivas. (Jiménez, 2009, p. 84)

Por lo anterior, el desarrollo de la DSN alteró la naturaleza de las instituciones militares. Se mezclaron y confundieron objetivos y competencias. Se produjo una distorsión negativa, "puesto que alteró los cánones profesionales y desvió los principios castrenses hacia funciones ajenas al quehacer militar" (Leal, 2006, pp.49-50).

Esta distorsión permitió que las instituciones castrenses vinculen a la población civil en la lucha contra el enemigo interno. Lo que Zelik denomina como "tercerización de la guerra" (2011, p.181), aludiendo al involucramiento de terceros o de seguridad privada en la confrontación. Así, por ejemplo, MicClintock (1992) citado en Colombia Nunca Más (2008), señala que en 1962 el General estadounidense Yarborug, director del Centro de Guerra Especial, visitó el país y emitió un informe secreto, el cual, entre otras cosas, establece que:

Debe crearse ahora mismo un equipo en el país acordado, para selecciona personal civil y militar con miras a un entrenamiento clandestino en operaciones de represión (...) Esta estructura se usará para presionar los cambios que sabemos, que se van a necesitar para poner en acción funciones de contra-agentes y contra-propaganda $y$, en la medida en que sea necesario, impulsar sabotajes y/o actividades terroristas paramilitares contra los conocidos partidarios del comunismo. (MicClintock, 1992, p. 222)

Finalmente, Leal (2006) posiciona la etapa de declinación en los años 80 y el fin de la Guerra Fría. Empero, en Colombia, aunque las instituciones castrenses 
aseguran que sus políticas ya no son sustentadas en la DSN, dinámicas y prácticas en el marco del conflicto político, social y armado denotan su vigencia y aplicación. La dicotomía amigo/enemigo parece persistir en el imaginario colectivo. De ahí la importancia de desplazar las lógicas heredadas de la DSN por nuevas visiones de seguridad que permitan deconstruir la noción militarista del término.

\section{Seguridad humana: nuevo referente de seguridad}

En el primer apartado se dijo que el fin de la Guerra Fría generó nuevos debates e interrogantes sobre el objeto referente de la seguridad. El debate permitió que se posicionaran nuevos paradigmas de seguridad. Møller (2000) denomina los nuevos referentes de seguridad como una "expansión del término", el cual se da como respuesta a tres interrogantes: 1) ¿Seguridad para quién? Tiene que ver con el objeto referente o con el elemento que debe protegerse. El Estado ya era insuficiente en el nuevo orden mundial. 2) ¿Seguridad para qué? Hace referencia a la ausencia de amenazas sobre ciertos valores, como los democráticos y sociales. Y 3) ¿Seguridad respecto a qué? Se trata de determinar las amenazas que se derivan de las nuevas dinámicas históricas y globales posguerra fría.

\section{Viraje hacia la seguridad humana}

En esta expansión del término se ubica la seguridad humana. Como se dijo, su antesala fue la Escuela de Copengahue y el sustento teórico son las teorías críticas de las Relaciones Internacionales que no se adscriben, directamente, a las teorías tradicionales (realismo, constructivismo, liberalismo o idealismo). Así, este nuevo paradigma posiciona como objeto referente a los seres humanos; el contenido son todas las dimensiones de la integridad y dignidad de los individuos.

La seguridad humana pretende, entonces, proteger a los seres humanos de todas las amenazas que atenten contra los seres humanos. Es una preocupación por las necesidades básicas y el desarrollo pleno de los individuos. Charles (2008) define la seguridad humana como "la protección de todos los individuos de las amenazas ambientales, económicas, alimentarias, médicas, personales y políticas" (p.122).

Charles David expone que algunas de las características del nuevo orden mundial que explican el surgimiento de este paradigma de seguridad son: difusión de valores democráticos, ascenso de los derechos humanos, influencia de organizaciones no gubernamentales, el impacto de los medios de comunicación durante crisis humanitarias o violaciones a los derechos humanos y desplazamiento de la guerra interestatal a la intraestal (2008, p. 127). 
De ahí que el posicionamiento de la seguridad humana es paralelo con la emergencia de los derechos humanos. Hamposn (2002), citado por Charles (2008, p.127), asume la seguridad humana como "una aspiración compartida de promover y proteger los derechos humanos, de tal manera que el bienestar y seguridad física de las personas puedan estar fuera de peligro."

Para el continente americano solo es hasta el año 2003 que se intenta desmilitarizar, al menos en teoría, la noción de seguridad y se reconoce de manera oficial e institucional la seguridad humana. La Conferencia especial sobre seguridad de la Organización de Estados Americanos realizada en México en octubre de 2003, plantea la necesidad de reconocer nuevos referentes de seguridad. Desde una influencia de la Escuela de Copenhague, en la declaración final alude a la seguridad multidimensional, reconociendo que, además de las amenazas tradicionales a los estados, existen nuevas amenazas: "Reconociendo que los Estados del Hemisferio enfrentamos tanto amenazas tradicionales a la seguridad como nuevas amenazas, preocupaciones y otros desafíos que por sus características complejas han determinado que la seguridad tenga un carácter multidimensional" (Organización de Estados Americanos, 2003 ).

De manera específica la declaración de la Conferencia en el numeral 4, literal e, reconoce la seguridad humana:

En nuestro Hemisferio, en nuestra condición de Estados democráticos comprometidos con los principios de la Carta de las Naciones Unidas y la Carta de la OEA, reafirmamos que el fundamento y razón de ser de la seguridad es la protección de la persona humana. La seguridad se fortalece cuando profundizamos su dimensión humana. Las condiciones de la seguridad humana mejoran mediante el pleno respeto de la dignidad, los derechos humanos y las libertades fundamentales de las personas, así como mediante la promoción del desarrollo económico y social, la inclusión social, la educación y la lucha contra la pobreza, las enfermedades y el hambre. (Organización de Estados Americanos, 2003)

Para el caso de Colombia será en el 2016, con la firma final de los acuerdos de La Habana, que se reconoce en documento vinculante esta noción.

\section{Características de la seguridad humana}

Esta concepción fue desarrollada por las Naciones Unidas en 1994 mediante el informe sobre Desarrollo Humano de 1994. El capítulo segundo se titula "nuevas dimensiones de la seguridad humana", donde se desarrolla las nociones de este nuevo paradigma. El informe parte de la necesidad de una transición de la seguridad nuclear a la seguridad humana, es decir, una seguridad cuya preocupación sea los seres humanos, su vida y dignidad. 
Establece el informe que "la seguridad humana se expresa en un niño que no muere, una enfermedad que no se difunde, un empleo que no se elimina, una tensión étnica que no explota en violencia, un disidente que no es silenciado" (PNUD, 1994, p. 25). El esfuerzo del Estado, en consecuencia, debe radicar en preservar la dignidad del ser humano en todas sus dimensiones. Por esto el informe señala como una de las características de la seguridad humana su carácter multidimensional, en la medida que aborda diferentes aristas que pueden vulnerar la vida y la dignidad de los individuos.

Por lo anterior, el informe de 1994 señala siete componentes de la seguridad humana:1) seguridad económica, 2) seguridad alimentaria, 3) seguridad en materia de salud, 4) seguridad ambiental, 5) seguridad personal, 6) seguridad de la comunidad y 7) seguridad política. Cada una de ellas como respuesta a diferentes amenazas que pueden verse expuestos los seres humanos. Adicional al carácter multidimensional, resalta el informe, estos componentes deben tener una interdependencia entre sí para garantizar de manera óptima y plena la seguridad humana.

Para Newnam (2001) existen tres interpretaciones de la seguridad humana. La primera, como necesidades básicas de los seres humanos, alimentación, cuidado personal, cultura etc. La segunda se basa en los límites de la seguridad estatal, mencionando que esta no siempre proporciona seguridad a los ciudadanos. La tercera sustenta la seguridad humana en el desarrollo social, resaltando la existencia de una relación directa entre seguridad y desarrollo social: mientras más elevado sea este último mayor será el grado de la primera.

Esta noción integral de seguridad, elevada a derecho en los acuerdos de La Habana, es un esfuerzo por desmilitarizar el concepto de seguridad. En especial, por desplazar la visión de seguridad instituida bajo la DSN. Este desplazamiento constituirá un avance significativo para la construcción de escenarios de paz. Sin detrimento de este avance, es necesario resaltar dos limitantes de la concepción de seguridad humana.

Primero, la pretensión holística del término conlleva a un ensanchamiento conceptual y a la dificultad de ponerlo en práctica con resultados concretos (Rivera, 2012, p. 41). Esto implica una difusa determinación de las amenazas y sus causantes. Las respuestas al interrogante de Møller resultan ambiguas, principalmente porque muchas de esas amenazas revisten una naturaleza estructural.

Segundo, el carácter antropocéntrico de la seguridad humana. Si bien la ubicación de los seres humanos posiciona una nueva agenda en seguridad, resulta limitada para abordar los derechos de la naturaleza o comunidades étnicas. El reto será posicionar una visión biocéntrica de seguridad que propenda por resguardar patrimonios naturales y culturales imprescindibles en la búsqueda de una paz completa. 


\section{Seguridad humana como herramienta para la construcción de paz}

Establecidos los elementos generales de la seguridad humana es necesario correlacionarlos con estudios de paz que permitan comprender la importancia de la adopción del término en los acuerdos de La Habana. Para ello se toma como referencia la categoría de paz positiva y paz negativa desarrolladas por Johan Galtung. Señala Moller (1996) que los aportes de Galtung sobre las concepciones de paz negativa/ positiva "podrían considerarse como precursores del emergente y amplio concepto sobre seguridad" (p. 770), entre ellos el de seguridad humana.

Los estudios de paz de Galtung se han ocupado de estudiar el tema no desde las aristas poder/seguridad. El foco de análisis es la paz donde la seguridad es un requisito para la construcción de paz positiva. Recordemos que para el pensador noruego existen tres formas correlacionadas de violencia en un conflicto armado: directa, estructural y cultural $(2003,2010,2016)$. La primera es el daño físico entre las partes en contiendas (muertes, heridos etc). La violencia estructural, la cual es invisible, responde a estructuras sociales, económicas y políticas que marginan a un sector poblacional. La violencia cultural, como ya se dijo, son imaginarios que legitiman los dos tipos de violencia.

Galtung (2010) establece que la paz positiva es aquella que se ocupa de las tres formas de violencia. Por el contrario, la paz negativa centra su preocupación en la violencia directa, es decir, es una paz que se asimila a la ausencia de confrontación o de guerra. Así, la paz positiva de Galtung implica, entre otras cosas, ocuparse de temas estructurales del ordenamiento social donde es transversal el bienestar y dignidad de los individuos: salud, educación, vivienda etc. Lo que exige a los Estados asumir las amenazas que atenten contra estos factores. Recordemos que para Newnam (2001), la seguridad humana depende del grado de desarrollo social, que no es otra cosa que abordar las estructuras socioeconómicas que excluyen a sectores de la población.

De ahí tenemos que, desde la seguridad humana, la paz no es solo la ausencia de guerra -ausencia de violencia directa-. Es la construcción de paz estructural y, de manera categórica, de paz cultural. Como se dijo, en Colombia la DSN deja profundas secuelas de violencia cultural. Luego, la seguridad humana debe constituirse como herramienta para deconstruir ese tipo de violencia.

\section{Conclusiones}

Los contextos de guerra han determinado el paradigma de seguridad. La segunda guerra mundial posicionó la seguridad del Estado y su soberanía. La Guerra Fría posicionó la noción de "seguridad nuclear", como garantía de los Estados para protegerse de las amenazas provenientes de otros Estados. La confrontación ar- 
mada en Colombia se enmarcó en la adopción de la DSN, trasladando las amenazas internacionales a la política doméstica.

Del marco explicativo se puede concluir -resaltando los componentes históricos y teóricos- que la seguridad nacional, en específico la DSN, se posiciona en la visión clásica de seguridad, responde al paradigma realista y nace en un momento histórico específico: la Guerra Fría. Por su parte, la seguridad humana se ubica en la vertiente expansiva de la noción de seguridad, se sustenta en teorías cítricas y es resultado de los cambios que implicó el fin de la guerra fría en el Sistema Internacional (Ver cuadro 1). En Colombia la seguridad humana emerge en un contexto en el que se busca la paz por medios pacíficos. De ahí que es posible concluir que mientras la DSN es hija de la guerra fría y dinamizadora de la confrontación interna; la seguridad humana irrumpe en el marco de un proceso de paz.

Es preciso aclarar que hasta ahora la seguridad humana que aparece en los acuerdos con categoría de derecho, es una alusión. Su realización dependerá del proceso de implementación de los acuerdos de La Habana, del proceso de paz en ciernes con el ELN y, en general, de la política de transición que demanda franquear el conflicto hacia la paz.

Tabla 1: Diferencias de paradigma clásico de seguridad y seguridad humana.

\begin{tabular}{|l|l|l|l|l|l|}
\hline $\begin{array}{c}\text { Paradigma } \\
\text { de seguridad }\end{array}$ & \multicolumn{1}{|c|}{$\begin{array}{c}\text { Modelo } \\
\text { seguridad }\end{array}$} & $\begin{array}{c}\text { Objeto } \\
\text { referente }\end{array}$ & $\begin{array}{l}\text { Teoría que } \\
\text { la sustenta }\end{array}$ & $\begin{array}{l}\text { Momento } \\
\text { histórico }\end{array}$ & Caso Colombia \\
\hline $\begin{array}{l}\text { Doctrina de } \\
\text { Seguridad } \\
\text { Nacional - } \\
\text { DSN-. }\end{array}$ & $\begin{array}{l}\text { Tradicional o } \\
\text { clásico }\end{array}$ & Estado & Realismo & $\begin{array}{l}\text { Guerra } \\
\text { fría }\end{array}$ & $\begin{array}{l}\text { Durante el } \\
\text { conflicto } \\
\text { armado }\end{array}$ \\
\hline $\begin{array}{l}\text { Seguridad } \\
\text { Humana }\end{array}$ & Expansivo & $\begin{array}{l}\text { Seres } \\
\text { Humanos }\end{array}$ & $\begin{array}{l}\text { Teoría } \\
\text { crítica }\end{array}$ & $\begin{array}{l}\text { Posguerra } \\
\text { fría }\end{array}$ & $\begin{array}{l}\text { Proceso de Paz } \\
\text { de La Habana. }\end{array}$ \\
\hline
\end{tabular}

Fuente: Elaboración propia.

La DNS militarizó el contenido de la seguridad y posicionó la lógica del enemigo interno. Estos dos factores permiten inferir que los principios de la DSN han sido dinamizadores del conflicto interno. A la par de la militarización, la dicotomía amigo/enemigo también se trasladó a la sociedad civil, convirtiéndose en un imaginario colectivo el "otro" que constituye amenaza. Luego, superar esa dicotomía es una condición sine qua non para superar la confrontación política, social y armada.

La alusión que hacen los acuerdos de La Habana a seguridad humana implica una ruptura con la visión clásica de seguridad. Se trata de un enunciado que trasciende la retórica discursiva, toda vez que tiene un contenido y un nuevo objeto referente: los seres humanos y su dignidad. A su vez, tiene una carga histórica, desplaza la noción de militarización como garantía de supervivencia. 
En síntesis, la seguridad después de la guerra demanda que el Estado llegue a territorios, no únicamente con fuerza pública, sino con instituciones sólidas que garanticen los derechos básicos. Hablar de paz implicará, entonces, que se hable de seguridad educativa, seguridad de salubridad, seguridad política, que permita ejercerla, vivirla y pensarla sin ser considerado enemigo. Seguridad para vivir dignamente como ciudadanos.

\section{Referencias}

Charles, P.D. (2008). La Guerra y la Paz, Enfoques Contemporáneos Sobre Seguridad y Estrategia. Barcelona: Icaria.

Colombia Nunca Más. (2008). Crímenes de Lesa Humanidad en la zona Quinta. Bogotá: Colectivo de Abogados José Alvear Restrepo.

Galtung, J. (2003). Violencia cultural. Gernika Gogoratuz, (14), pp. 1-31

Galtung, J. (2010). Direct, Structural, and Cultural Violence. The Oxford International Encyclopedia of Peace, 1 (4), pp. 312-316.

Galtung, J. (2016). La violencia cultural, estructural y directa. Cuadernos de estrategia, (168), pp. 147-168.

Hobsbawm, E. (1994). Historia del siglo XX. Bogotá: Planeta

Hobsbawm, E. (2007). La Guerra y la paz en siglo XXI. Barcelona: Crítica.

Jiménez, C. (2009). Aplicación e Instrumentalización de La doctrina de seguridad Nacional en Colombia (1978-1982): efectos en Materia de derechos Humanos. Revista Colección, XV (20), pp. 75-105.

Leal, F. (2003). La Doctrina de Seguridad Nacional: Materialización de la Guerra Fría en América Del Sur. Revista de Estudios Sociales, I (15), pp. 74-87. doi: https:// doi.org/10.7440/res15.2003.05

Leal, F. (2006). La inseguridad de la seguridad. Bogotá: Planeta.

Møller, B. (2000). Seguridad Nacional, Societal y Humana: El marco general y el caso de los Balcanes. Fasoc, 15 (4), pp. 4-15. Recuperado de: http://biblioteca.cejamericas.org/bitstream/handle/2015/3405/Art.pdf?sequence=1\&isAllowed=y

Møller, B. (1996). Conceptos sobre seguridad: nuevos riesgos y desafíos. Desarrollo Económico, 36, pp.769-792.

Morgenthau, H. (1986). Política entre las naciones: La lucha por el podery la paz. Buenos Aires: Editor Latinoamericano.

Newman, E. (2001). Human Security and Constructivism. International Studies Perspectives Journal, 2 (3), pp. 239-251. doi: 10.1111/1528-3577.00055 
Organización de Estados Americanos. (2003). Acta final de la conferencia especial sobre seguridad. Recuperado de: http://www.oas.org/csh/ces/

Orozco, G. (2006). El concepto de la seguridad en la Teoría de las Relaciones Internacionales. Revista CIDOB d'Afers Internacionals, (72), pp.167-180.

Orozco, G (2006a). El aporte de la Escuela de Copenhague a los estudios de seguridad. Revista Fuerzas Armadas y Sociedad, (1), pp. 141-162.

Oswald, U. \& Gunter, H. (2010). Globalización y desafíos ambientales cambian la conceptualización de seguridad en América Latina. En U. Oswald \& H. Gunter (Eds.), Reconceptualizar la seguridad en el siglo XXI (pp. 37-70) México: UNAM

PNUD (1994). Informe de desarrollo humano. México: Fondo de cultura Económica.

Ramírez, J. (2010). Thomas Hobbes y el Estado absoluto: del Estado de razón al Estado de terror. Medellín: Universidad de Antioquia.

Rivera, F. (2012). La seguridad Perversa. Política democracia y derechos humanos en Ecuador 1998-2006. Quito: Flacso.

Schmitt, C. (1999). El Concepto De Lo Político. Madrid: Alianza Editorial

Vergara, J. (2005). Doctrinas de Seguridad Nacional. Conceptos Fundamentales. Pensamiento crítico Latinoamericano. Santiago de Chile: Universidad Católica Silva Henríquez.

Vargas, A. (2009). Es posible y deseable la solución del conflicto interno armado hoy?. En A. Vargas. (Ed.), Colombia: escenarios posibles de Guerra y Paz (pp. 19-92). Bogotá: Universidad Nacional.

Vargas, A. (2010). Seguridad en democracia: un reto a la violencia en América Latina. Buenos Aires: Consejo Latinoamericano de Ciencias Sociales - CLACSO.

Wæver, O. (2010). Paz y Seguridad: dos conceptos en evolución y su relación cambiante. En U. Oswald \& H. Gunter. (Eds.), Reconceptualizar la seguridad en el siglo XXI (pp. 71-100). México: UNAM.

Wendt, A. (1992). Anarchy is what state make of it: the social construction of power politics. International Organization, 46 pp. 391-425.

Zelik, R. (2011). La guerra asimétrica. Una lectura crítica de la transformación de las doctrinas militares occidentales. Estudios políticos (39), pp.168-195. 